\title{
21
}

\section{Curve Numbers in Stormwater Runoff Simulation}

Ivan Muzik

Urban runoff models require the determination of excess rainfall for pervious areas in catchments. This is generally achieved by means of infiltration equations such as, for example, the Horton and Green-Ampt equations in the SWMM and MIDUSS models. Some models (MIDUSS, OTTHYMO) also use the SCS runoff curve number method. Computation of the excess rainfall by any of these methods requires the estimation of two or three parameters which are generally functions of the land use and soil properties, including the moisture content of the pervious areas. Values of the parameters can be obtained by infiltrometer measurements, but field measurements are time-consuming and may not be practical.

Typical parameter values are available in the literature but, in many cases, modelers use optimization techniques to estimate the "best" parameter values during the calibration of the model. Ideally, the parameter optimization should be guided by parameter values derived for the specific catchment from observed rainfall-runoff data, but independently of the calibration process. In this chapter an asymptotic method of estimating the curve number $C N$ and initial abstraction $I_{a}$ for a watershed, from observed rainfall and runoff events, is presented.

The proposed method is asymptotic in the sense that the estimated values approach the "true" values as the number of observations increases. The method provides estimates of spatially averaged $C N$ and $I_{a}$ for each event for the catchment area upstream of the streamflow gauging station.

Muzik, I. 2003. "Curve Numbers in Stormwater Runoff Simulation." Journal of Water Management Modeling R215-21. doi: 10.14796/JWMM.R215-21.

(C) CHI 2003 www.chijournal.org ISSN: 2292-6062 (Formerly in Practical Modeling of Urban Water Systems. ISBN: 0-9683681-7-4) 


\subsection{Introduction}

\subsubsection{Background}

The Soil Conservation Service (SCS) procedure for computing abstractions from storm rainfall (National Engineering Handbook, 1985) relates the depth of excess rainfall $P_{e}$ to the total rainfall depth $P$, initial abstraction $I_{a}$, and potential maximum retention $S$ by the following equation:

$$
P_{c}=\frac{\left(P-I_{a}\right)^{2}}{P-I_{a}+S}
$$

An empirical relation suggested by the Soil Conservation Service:

$$
I_{a}=\lambda S
$$

with $\lambda=0.2$, is commonly applied to simplify Equation 21.1 into:

$$
P_{e}=\frac{(P-0.2 S)^{2}}{P+0.8 S}
$$

However, values of $\lambda$ varying in the range from 0.0 to 0.38 have been documented in a number of studies encompassing various geographical locations (Springer et al. 1980, Cazier and Hawkins 1984, Bosznay 1989, Ponce and Hawkins 1996). The second power to which $I_{a}$ is raised in Equation 21.1 makes the calculation of $P_{e}$ highly sensitive to errors in $I_{a}$. Reduction of uncertainty in estimated $I_{a}$ values can thus lead to much improved estimates of the excess rainfall depth $P_{e}$.

The potential maximum retention $S$, which varies in the range $0 \leq S \leq \infty$, is assumed to depend on the soil type, land use, land cover and the antecedent moisture condition. It is high for deep sandy soils and low for shallow clayish soils. For convenience, the potential maximum retention $S$, in $\mathrm{mm}$, is converted into a parameter $C N$, having a range of values between 0 and 100 , by the following equation:

$$
C N=\frac{25,400}{S+254}
$$

The parameter $C N$, called the curve number, is equal to zero $(S=\infty)$ when there is no direct runoff generated. The value of 100 , on the other hand, means no abstractions $(S=0)$, and the depth of excess rainfall equals the depth of total 
rainfall. For practical application, curve numbers have been tabulated by the Soil Conservation Service (SCS) on the basis of generalized classes of soil type and land use and for three discrete antecedent moisture condition classes.

\subsubsection{Objectives}

An important process in modeling surface runoff is the generation of excess rainfall. The computation of excess rainfall by an infiltration model depends on parameters that are uncertain and difficult to measure. The SCS runoff curve model requires the estimation of two parameters, $S$ and $I_{a}$, respectively. The accuracy and reliability of the estimates of these parameters can be improved, prior to catchment model calibration through parameter optimization, by direct analysis of the catchment observed rainfall and runoff data.

The objectives of this study were to:

1. develop a method of estimating the catchment $S$ and $I_{a}$ from observed rainfall and runoff data, and

2. evaluate the applicability and performance of the proposed method under different conditions.

\subsection{Methodology}

\subsection{1 $S$ vs $I_{a}$ Relationship}

Equation 21.1 is the SCS model for converting the cumulative catchment rainfall $P$ into the cumulative catchment excess rainfall $P_{e}$. The problem is to identify the model parameters $S$ and $I_{a}$ given a sufficient number of observations of $P$ and the corresponding $P_{e}$ values. The value of $P$ represents the average catchment rainfall depth for a storm event. The value of $P_{e}$ can be determined by separating baseflow from the observed outflow hydrograph, and dividing the direct runoff volume by the catchment area.

For a given pair of observed values $\left(P, P_{e}\right)$, Equation 21.1 can be rewritten to solve for $S$, yielding:

$$
S=\frac{\left(P-I_{a}\right)^{2}}{P_{e}}-\left(P-I_{a}\right)
$$

The $S$ vs $I_{a}$ relationship plots as the curve shown in Figure 21.1. The curve has a minimum at $S=-P_{e} / 4$ and $I_{a}=P-P_{e} / 2$, determined from the requirements for the minimum, given by: 
and

$$
\frac{d S}{d I_{a}}=\frac{2}{P_{e}}\left(I_{a}-P\right)+1=0
$$

$$
\frac{d^{2} S}{d I^{2}}=\frac{2}{P_{e}}>0
$$

However, the only physically meaningful part of the curve, shown by a thick line in Figure 21.1, is delimited by the condition that $S S \geq 0$ and $I_{a} \geq 0$. For a given $P, P_{e}$, the potential retention $S$ reaches its maximum value when $I_{a}=0$, given by:

$$
S_{\max }=\frac{P^{2}}{P_{e}}-P
$$

Conversely, when $S=0$, the initial abstraction $I_{a}$ becomes maximum, equal to:

$$
I_{a(\sin \alpha)}=P-P_{e}
$$

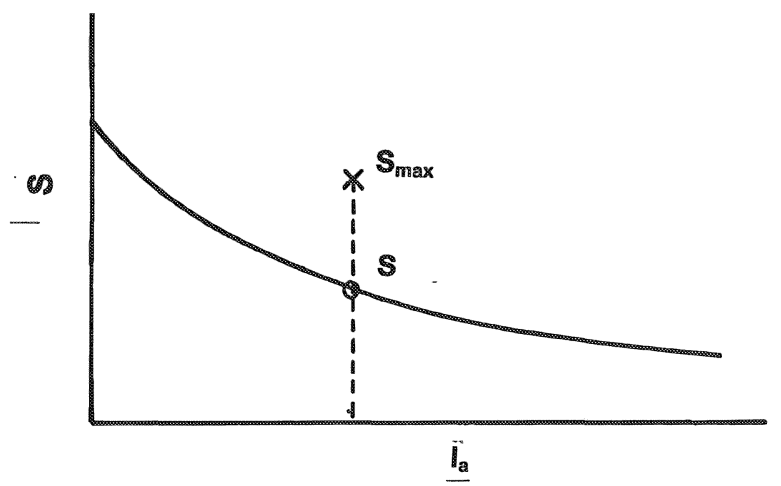

Figure 21.1 S $v s \mathrm{I}_{\mathrm{a}}$ relationship for an event with identified $P$ and $P_{e}$. The actual potential retention for the event $S,\left(0 \leq S \leq S_{\max }\right)$, is hypothesized to be a function of $P 5$

\subsubsection{Estimation of $\mathrm{S}$}

The potential maximum retention $S$ depends, as stated previously, on the soil type, land use/cover complex and the antecedent soil moisture condition (AMC). Assuming that the first two factors remain relatively unchanged for a 
catchment, at least on a seasonal basis, the most dynamic and dominant factor influencing the variation of $S$ between storm events is the AMC. The SCS method recognizes three discrete classes of AMC. The standard curve numbers given in the SCS tables correspond to AMC 2, which is generally assumed to represent a typical design situation. A choice of AMC 1 results in lesser runoff depth, whereas greater runoff results from a choice of AMC 3 . The level of AMC is based on the total 5-day antecedent rainfall P5, for dormant and growing season (National Engineering Handbook, 1985).

In this chapter a continuous relationship between $S$ and $P 5$ is developed. The curve representing this relationship should have a shape similar to that shown in Figure 21.2, reflecting the hypothesis that $S$ should be decreasing from the initial "dry" value in an exponential-like fashion, as $P 5$ increases from zero.

The proposed method for the derivation of the empirical S-P5 curve for a catchment requires that $P, P_{e}$ and $P 5$ data be available for a relatively large number of events. The potential maximum retention $S$, given by Equation 21.5), cannot be directly calculated because the values of $I_{a}$ are not readily measured and thus are generally unknown. However, assuming $I_{a}=0$, Equation 21.5 reduces to Equation 21.8, which enables the calculation of $S_{\max }$ for each event from the available data. The $S_{\max }$ values will plot above the "true" $S$ - $P 5$ curve, as illustrated in Figure 21.2. This is because if the actual $I_{a}$ is greater than zero, the actual $S<S_{\max }$, as can be seen from Figure 21.1. It is assumed that if a large sample of data is analyzed, there should be a sufficient number of events for which $I_{a}$ approaches zero. This would then allow one to draw an enveloping

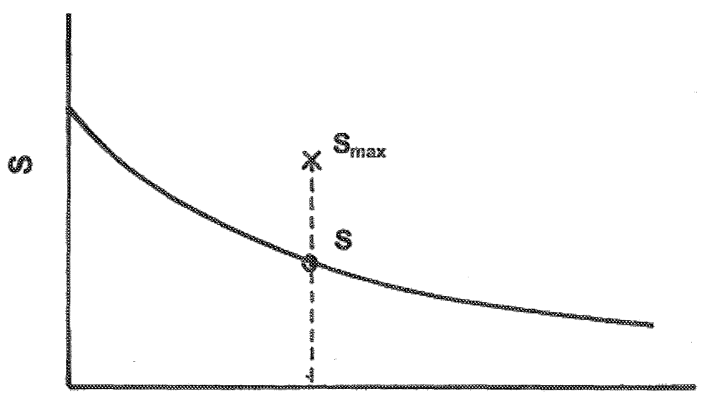

P5

Figure 21.2 Hypothesized $S$ vs $P 5$ relationship. $S_{\max }$ and $S$ are the maximum possible and the actual potential retention, respectively, for an event with identified $P 5, P, P_{e}$ and $I_{a}$. 
curve from below the plotted $S_{\max }$ vs $P 5$ values, which is an estimation of the "true" $S$ vs $P 5$ curve. The estimation gets progressively better as the number of analyzed events increases.

\subsubsection{Estimation of $I_{a}$}

The fitted $S$ vs $P 5$ curve is used to estimate the value of $S$ for each event in the data sample, that is, for each event the values of $S, P$ and $P_{e}$ are known. The event initial abstraction $I_{a}$ can now be computed by solving Equation 21.1 for $I_{a}$, yielding:

$$
I_{a}=P-\frac{P_{e}+\left(P_{e}^{2}+4 P_{e} S\right)^{1 / 2}}{2}
$$

Only the positive sign in the numerator of Equation 21.10 has physical meaning, because the negative sign would always yield $I_{a} \geq P$, which is not possible.

The usual physical interpretation of the initial abstraction (Mishra and Singh, 1999) is that it is the sum of the interception, depression storage and infiltration before surface runoff occurs. These terms do not explicitly appear in Equation 21.10, but could be considered as implicitly included in terms $P_{e}$ and $S$. However, because of this fuzzyness, it is very difficult to determine the $I_{a}$ values independently.

\subsection{Results}

\subsubsection{Study Region}

The outlined method of determining the $S$ vs $P 5$ curve for a watershed requires a large number of rainfall and runoff observations, covering an extensive range of AMCs in terms of the P5 values. This data requirement necessitated a regional approach to verify the method. 31 watersheds with a total of 61 observed events were selected. The watersheds are located along the Alberta foothills. Land cover is predominantly coniferous or mixed forest and the soils belong to the $\mathrm{B}$ or $\mathrm{C}$ hydrologic soil group, according to the SCS classification. The average $C N$ for the region, determined from standard SCS tables (AMC 2), is 76 , ranging from 74 to 81 . 


\subsubsection{Analysis Results}

For each of the 61 events, the 5-d antecedent precipitation $P 5$ and the average total rainfall $P$ were calculated by the Thiessen polygon method. The depth of excess rainfall $P_{e}$ was then computed by separating baseflow from the total runoff hydrograph, using the variable slope method (Chow et al., 1988). With $P$ and $P_{e}$ known, $S_{\max }$ was calculated from Equation 21.8. Figure 21.3 shows a semi-log graph of the computed $S_{\max }$ values plotted versus $P 5$, and the estimated $S$ vs $P 5$ relationship given by the segmented line drawn by eye below the plotted data.

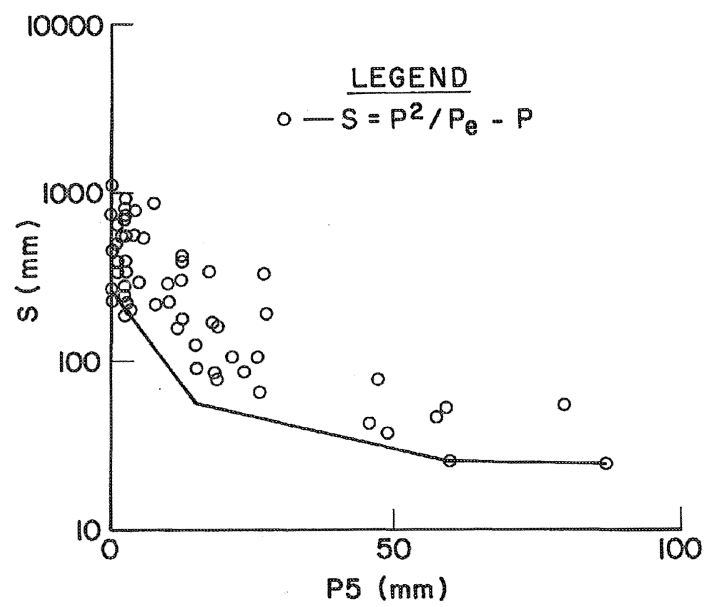

Figure 21.3 Estimated $S$ vs $P 5$ relationship (segmented line) for the study region.

Estimates of $\mathrm{S}$ valued for each of the 61 analyzed events were obtained as a function of P5, represented by the segmented line in Figure 21.3. The initial abstraction values $I_{a}$ were then computed by Equation 21.10. An attempt was made to find a regression equation for $I_{a}$, based on measurable parameters such as $P, S$ and the season of the year, but without much success. The highest coefficient of determination obtained was $r^{2}=0.52$.

When the initial abstraction is assumed to be a random variable it was found for the study region that the log-Pearson 3 distribution provided a good fit to $I_{a}$ values divided into two groups, according to whether $P 5<30 \mathrm{~mm}$, or $P \geq 30 \mathrm{~mm}$. The fitted distributions are shown in Figure 21.4.

The computed initial abstraction ratio $\lambda=I_{d} / S$ varied in the range $0.0 \leq \lambda$ $\leq 0.39$ with the average value equal to 0.093 . For watersheds having sufficient 


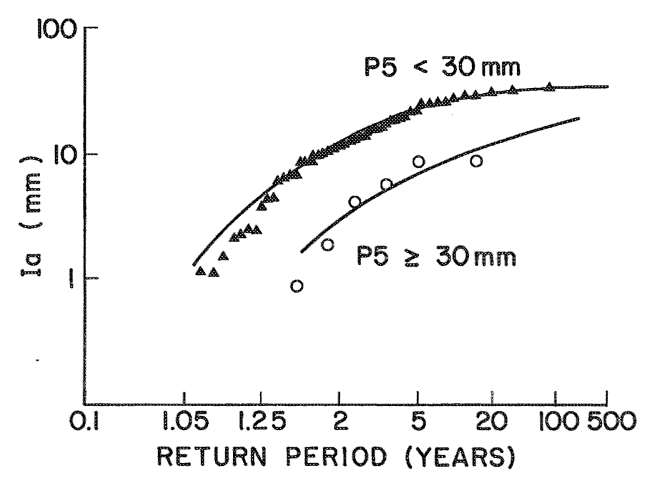

Figure 21.4 Computed $I_{a}$ values for the study region fitted by log-Pearson 3 distributions.

land use and soil type information the runoff curve numbers were determined from standard SCS tables, and adjusted to the AMC as follows (Chow et al., 1988):

$$
\begin{aligned}
& \text { AMC 1: } P 5<36 \mathrm{~mm} \\
& \text { AMC 2: } 36 \mathrm{~mm} \leq P 5 \leq 53 \mathrm{~mm} \\
& \text { AMC 3: } P 5>53 \mathrm{~mm}
\end{aligned}
$$

The average $C N$ values for each AMC class are shown in Table 21.1 in comparison with the averages derived from observations by the proposed method. The tabulated values are lower for all three AMC classes than the derived values. The tendency of the standard SCS runoff curve procedure to underestimate the runoff volume has been reported by other researchers (Hiemstra and Reich, 1967; Bales and Betson, 1981).

Table 21.1 Average tabulated and derived $\mathrm{CN}$ values.

\begin{tabular}{cccc}
\hline AMC & No. of Events & Tabulated CN & Derived CN \\
\hline 1 & 14 & 57 & 61 \\
2 & 2 & 76 & 88 \\
3 & 3 & 88 & 91 \\
\hline
\end{tabular}

\subsection{Discussion and Summary}

The runoff curve number method for the estimation of direct runoff from storm rainfall is well established in hydrologic engineering. Its popularity is rooted in its convenience, its simplicity, its authoritative origins, and its responsiveness 
to four readily grasped catchment properties: soil type, land use/treatment, surface condition, and antecedent condition (Ponce and Hawkins, 1996).

The method is simple only if it is assumed that the initial abstraction ratio $\lambda=0.2$ (or some other constant value), and the standard SCS tables are used to determine the $C N$ value. However, values of $\lambda$, reported in the literature and in the present chapter, vary in the range $0 \leq \lambda \leq 0.39$. Because the runoff curve number method is sensitive to initial abstraction $\left(I_{a}=\lambda S\right)$, estimation of runoff curve numbers directly from local rainfall and runoff data can be expected to increase the accuracy of the method.

The present chapter proposes a method for estimation of runoff curve numbers from measured data. The method requires assembly of corresponding sets of rainfall-runoff data encompassing a wide range of antecedent moisture conditions. For each event the total rainfall depth $P$ and the excess rainfall depth $P_{e}$ are identified. Analysis of the runoff curve number equation shows that for a given set of $P, P_{e}$ values, the potential maximum retention $S$ is functionally related to the initial abstraction $I_{a}$ and varies in the range $0 \leq S \leq P^{2} / P_{e}-P$. The proposed methodology assumes that the value of $S$ in this range depends on the antecedent moisture condition, indicated by a surrogate variable $P 5$, the $5-\mathrm{d}$ antecedent rainfall depth. The corresponding range for the initial abstraction is $0 \leq I_{a} \leq P-P_{e^{\circ}}$.

There is no independent relationship available to evaluate $I_{a}$, thus, for each data set $P, P_{e}$ there are two unknowns, $I_{a}$ and $S$, in the runoff curve number equation. According to the proposed methodology, $S$ can be calculated from the data by assuming $I_{a}=0$. This is the maximum $S$ possible for a given set $P, P_{e}$, and can be plotted against $P 5$. It is postulated that, if there is a sufficient number of such cases in the data, i.e. $I_{a} \rightarrow 0$, it is possible to draw a curve that envelopes the plotted values $S$ vs $P 5$ from below, which approximates the true $S$ vs $P 5$ relationship. The estimated $S$ for each event can then be used to calculate the corresponding $I_{a}$.

The proposed methodology was applied to 61 events observed on 31 watersheds. The watersheds were selected to form a relatively homogeneous region representing a typical forested foothills watershed in Alberta. The results confirmed the feasibility of the method. The computed $C N$ values were realistic and higher than those determined from tables and assuming $I_{a}=0.2 S$. The computed initial abstraction values appeared to be random and varied in the range $0 \leq I_{a} \leq 0.39$. The results lead to the following comments:

1. In this study, $I_{a}$ was allowed to become zero. Future studies may consider a greater than zero minimum value of $I_{a}$ as physically more appropriate. 
2. The 5-d antecedent rainfall may not be the correct parameter for all types of watersheds. A weighted average rainfall according to the time of rainfall occurrence during the $5-\mathrm{d}$ period may be more appropriate.

3. Determination of the event rainfall depth $P$ and the 5-d antecedent rainfall $P 5$ may be potentially subject to large errors, depending on the number of raingauges available and the method used to calculate the watershed average rainfall, and the spatial rainfall variability during the event.

\section{References}

Bales, J. and Betson, R.P. (1981). "The curve numbers as a hydrologic index." Singh, V.P. (Editor), Rainfall-Runoff Relationship, Water Resources Publications, Littleton, CO 80161, USA, pp. 371-386.

Bosznay, M. (1989). "Generalization of SCS curve number method." Journal of Irrigation and Drainage Engineering, 115(1):139-144.

Cazier, D.J and Hawkings, R.H. (1984). "Regional application of the curve number method." Water Today and Tomorrow, Proc., ASCE Irrig. and Drain. Div. Spec. Conf., ASCE, New York, NY, USA.

Chow, V.T., Maidment, D.R. and Mays, L.W. (1988). Applied Hydrology, McGrawHill, New York, NY, USA.

Hiemstra, L.V. and Reich, B.M. (1967). "Engineering judgment and small area flood peaks." Hydrology Paper 19, Colorado State University, Fort Collings, CO, USA.

Mishra, S.K. and Singh, V.P. (1999). "Another look at SCS-CN method". Journal of Hydrologic Engineering, Vol. 4, No. 3, pp257-264.

"National Engineering Handbook, Section 4, Hydrology" (1972). United States Department of Agriculture, Soil Conservation Service, United States Government Printing Office, Washington, DC, USA.

Ponce, V.M. and Hawkins, R.H. (1996). "Runoff curve number: Has it reached maturity?" Journal of Hydrologic Engineering, Vol. 1, No. 1, pp. 11-19.

Ponce, V.M. (1989). Engineering Hydrology Principles and Practices. Prentice Hall, Englewood Cliffs, NJ, USA.

Springer, E.P., McGurk, B.J., Hawkins, R.H. and Coltharp, G.B. (1980). "Curve numbers from watershed data." Proc., Symposium on Watershed Management, ASCE, Boise, ID, USA, pp. 938-950. 